\title{
Mechanical Response of Beams of a Nonlinear Viscoelastic Material
}

\author{
ALAN WINEMAN and RAYMOND KOLBERG* \\ Department of Mechanical Engineering and Applied Mechanics \\ University of Michigan \\ Ann Arbor, Michigan 48109
}

\begin{abstract}
A constitutive equation for nonlinear viscoelasticity is used to model the mechanical response of solid polymers such as polycarbonate. The nonlinearity arises from a reduced time which causes stress relaxation to accelerate with increasing strain. The constitutive equation can account for the occurrence of yield in a homogeneous uniaxial constant strain rate test. The constitutive equation is used in a study of the pure bending of beams. It is assumed that the classical assumption of beam theory is valid, i.e., plane sections remains plane. At each fixed time, the strains vary linearly through the depth of the beam. At a fixed material element the strain varies in time with the curvature. This spatial variation of the strains combined with the nonlinear dependence of the reduced time on strain leads to a significantly different response from that given by traditional beam theory. The implications of this for the bending moment history, stress distributions, and other factors that relate to beam design are discussed.
\end{abstract}

\section{INTRODUCTION}

$\mathrm{S}^{\mathrm{t}}$ tructural components using polymers are often designed by treating the materials as though they exhibit linear elastic or linear viscoelastic response. The behavior of commonly used unfilled amorphous polymers, such as polycarbonate, differs significantly from linear elastic or viscoelastic response near the material yield point. Thus, designs may be conservative so that yield is avoided, or they do not take full advantage of the available material response.

A number of authors have recently studied a constitutive equation for the nonlinear viscoelastic response of polymers such as polycarbonate and have shown that it can account for yield. The dominant property of this constitutive equation is that a strain induced increase in fractional free volume causes the stress relaxation process to accelerate. Using this constitutive equation in numerical simulations of constant extension rate experiments, Shay and Caruthers $(1,2)$ demonstrated that yield could be calculated. While the results of their calculations are in only qualitative agreement with experimental results, it is clear that the constitutive equation, with appropriate selection of material parameters, is capable of an accurate simulation of yield. Knauss and Emri $(3,4)$ determined a set of material properties for polyvinyl acetate for this constitutive equation and showed that it simulated yield under constant strain rate conditions. Wineman and Waldron (5) considered a form of this constitutive equation in which general strains could cause a acceleration of stress relaxation. They proved that such a model can predict yield under a variety of strain or stress control histories.

Shay and Caruthers $(1,2)$ and Knauss and Emri $(3,4)$ applied this constitutive equation to conditions in which the strains and stresses do not vary spatially. Wineman and Waldron (5) presented an example in which there is spatial variation of strains and stresses. They considered a hollow cylinder composed of a nonlinear viscoelastic material. The cylinder is fixed at its inner surface and its outer surface is subjected to a small amplitude time dependent rotation about the cylindrical axis. This causes each material element to undergo a one dimensional shear strain history. Waldron and Wineman (5) used a constitutive equation for the nonlinear viscoelastic shear response which is of the same form as that discussed above. They showed that the shear stress decreases monotonically with radius at each instant and, hence, so do the shear strain and rate of stress relaxation. This interaction of strain accelerated stress relaxation and spatially varying strain leads to interesting new phenomena. For example, a localized region of rapidly growing shear strains can evolve near the inner surface. There can also be a yield like relation between the applied moment and the rotation of the outer surface.

This constitutive equation is applied here to the study of polymeric beams. It will be shown that, as a

\footnotetext{
* General Electric Plastics.
} 


\section{A. Wineman and R. Kolberg}

consequence of the strain variation through the beam, the moment, curvature or stress distribution histories will differ significantly from those obtained by assuming the material to be linear elastic or viscoelastic. These results have interesting consequences for the design of structural components.

The constitutive equation is introduced in the next section and the equations for pure bending are developed in the following section. The formulation is restricted to conditions of geometric linearity (small strains and rotations) even though the material response is nonlinear. Only pure bending is considered, so that attention can be focused on variation of stresses and strains through the depth of the crosssection. Two different deformation control histories are studied. The fourth section is concerned with a step change in curvature. The fifth section contains results for constant curvature rate histories. Even with these assumptions, it is found that there are a number of interesting consequences of the material behavior. For this reason, moment control histories will be considered in a later study.

\section{CONSTITUTIVE EQUATION}

A basic assumption in the analysis of pure bending is that each material element is in a uniaxial stress state. For such states, the constitutive equation under consideration reduces to the form

$$
\sigma(t)=\varepsilon(0) G[\xi(t)]+\int_{0}^{t} G[\xi(t)-\xi(s)] \frac{d}{d s} \varepsilon(s) d s
$$

in which $\sigma(t)$ is the normal stress at the current time $t, \varepsilon(s), 0 \leq s \leq t$, is the uniaxial strain at time $s$ in the direction of the stress, and $G(t)$ is the stress relaxation function of linear viscoelasticity in uniaxial extension. $\xi(s)$ is a time llike variable which is expressed in terms of the strain history by the relation

$$
\xi(s)=\int_{0}^{s} \frac{d x}{\phi(\theta(x))}
$$

$\theta(x)$ denotes the volumetric strain at time $x$, and $\phi(\theta)$ is another material property, usually related to the time-temperature shift function. It is a positive, monotonically decreasing function of $\theta . \xi(s)$ is often referred to as the reduced or pseudo time.

The material property functions $G(t)$ and $\phi(\theta)$ are chosen, for the purposes of the numerical example presented here, to represent properties of a typical material rather than of a specific material. The stress relaxation function is taken to have the form

$$
G(t)=G_{o}\left[(1-g) e^{-t / \tau_{0}}+g\right]
$$

in which $G_{o}=G(0), g=G_{x} / G_{0}$, where $G_{0}=\lim G(t)$ as $t \rightarrow \infty$, and $\tau_{0}$ is a characteristic relaxation time. $\phi(\theta)$ is given by

$$
\log _{10} \phi(\theta)=\frac{B}{2.303}\left[\frac{1}{f(\theta)}-\frac{1}{f(0)}\right]
$$

where $B$ is a material constant and $f(\theta)$ is given by

$$
f(\theta)=f_{o}+C|\theta|
$$

in which $C$ is a constant and $f_{0}=f(0)$. This expression is that used by Knauss and Emri (4) with the additional assumption that $f(\theta)$ depends only on the magnitude of the volumetric strain. It implies that the response in uniaxial extension will be the same as in uniaxial compression. With this assumption, it is easier to illustrate the consequences of the interaction between the strain accelerated stress relaxation and the spatial variation of the strain. The implications of different response in extension and compression will be discussed later.

When $C=0$, the constitutive equation reduces to that of linear viscoelasticity. In the numerical results presented in the fourth and fifth sections $C=0.05$ and $g=0.001$, which are the values used by Waldron and Wineman (5). Also, $B=0.474$ and $f_{0}=0.0217$, which are the values chosen by Knauss and Emri (4).

The calculation of $\theta(x)$ requires knowledge of the Poisson's ratio function of linear viscoelasticity. It is assumed that the Poisson ratio function varies sufficiently slowly with $t$ that it can be approximated as a constant. This has the benefit of simplifying the computations while still making it possible to illustrate the consequences of the interaction between the strain accelerated stress relaxation and the spatial variation of the strain. Then in Eq $2 \theta(x)$ can be taken as $\varepsilon(x)$, and the constant $C$ can be reinterpreted as including Poisson's ratio.

\section{PURE BENDING}

Consider a straight beam whose cross section has a line of symmetry, as shown in Fig. 1 . Let the $x$-axis of a coordinate system be along the line which is normal to the cross section and which passes through its centroid and let the $y$-axis be along the line of symmetry of the cross section.

The standard assumptions are made about the bending deformation and apply at each time $t$. The deformation is sufficiently small that the change in

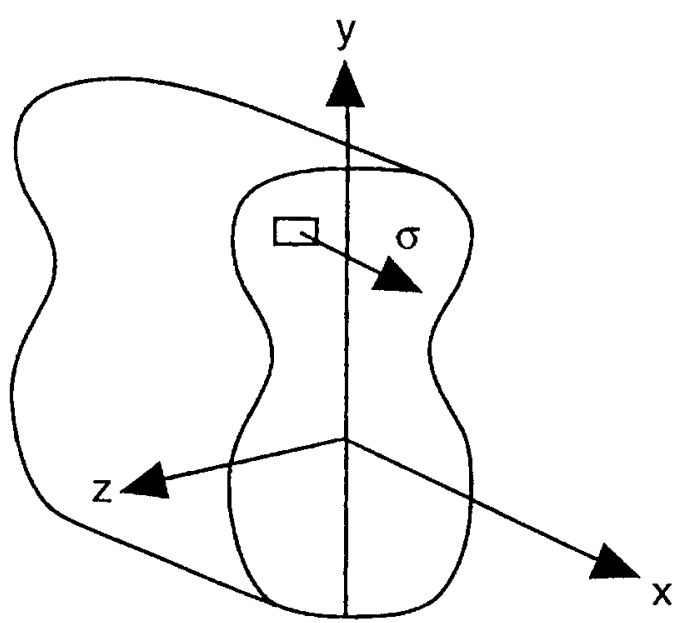

Fig. 1. Cross section and coordinate axes. 
the shape of the cross-section can be neglected. Each plane cross section rotates about an axis in its own plane parallel to the $z$-axis and remains plane. Line segments which are initially straight and parallel to the $x$-axis deform into circular arcs, and either elongate or shorten. Figure 2 shows the deformed shape at a typical time $t$. The coordinate of the neutral axis and its radius are denoted, respectively, by $d(t)$ and $\rho(t)$. The usual development leads to the following expression for the normal strain of the line at coordinate $y$ at time $t$,

$$
\varepsilon(y, t)=[d(t)-y] K(t)
$$

in which $K(t)=1 / \rho(t)$ and is the curavture. According to $E q 6$, the normal strain varies linearly through the cross-section at each instant, and the location of the neutral axis may vary with time.

By Eqs 1 and 2, the stress $\sigma(y, t)$ on an area increment of the cross section at coordinate $y$ is expressed in terms of the strain history $\varepsilon(y, s), 0 \leq$ $s \leq t$, by

$$
\begin{aligned}
\sigma(y, t)= & \varepsilon(y, 0) G[\xi(y, t)] \\
& +\int_{0}^{t} G[\xi(y, t)-\xi(y, s)] \frac{\partial}{\partial s} \varepsilon(y, s) d s
\end{aligned}
$$

where

$$
\xi(y, s)=\int_{0}^{s} \frac{d x}{\phi(\varepsilon(y, x))}
$$

As seen from $E q 4, \phi$ is nonlinear in the strain. Consequently, the stresses do not vary linearly in $y$.

Let there be no normal force on the cross-section at each time $t$. This requires that

$$
\iint_{A} \sigma(y, t) d A=0
$$

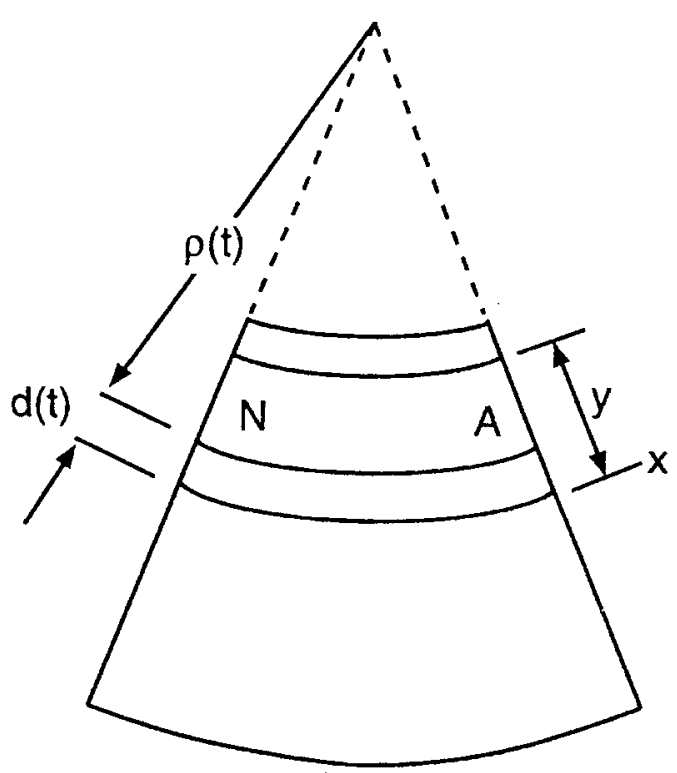

Fig. 2. Geometry of deformation. in which the integration is taken over the undeformed shape of the cross section. Given a curvature history, $E q 9$ becomes an equation for $d(t)$, the coordinate of the neutral axis. Let $M(t)$ denote the bending moment on the cross section acting about the $z$-axis at time $t$. Then

$$
M(t)=-\iint_{A} y \sigma(y, t) d A
$$

The implications for beams of the material response represented by the constitutive equation are most easily appreciated by considering the following special case. Let the line $y=0$ be an axis of symmetry of the cross section and recall that the material has the same response in tension and compression. Then $E q 9$ is satisfied with $d(t)=0$, so that the neutral axis coincides with the centroid at all times $t$. To see this, let $d(t)=0$ in Eq. 6. From Eqs 4, 5, and 8 it can be seen that $\xi(y, s)=\xi(-y, s)$. Equations 6 and 7 then imply that $\sigma(y, t)=-\sigma(-y, t)$. By the symmetry of the cross-section about the line $y=0$, it follows that $E q 9$ is satisfied. With this result, $E q 6$ reduces to

$$
\varepsilon(y, t)=-y K(t)
$$

\section{STEP CHANGE IN CURVATURE}

Let the beam be subjected to a deformation in which the curvature is instantaneously changed from $K(t)=0$ when $t<0$ to $K(t)=K_{o}$ for $t \geq 0$. By Eq 11 , $\varepsilon(y, t)=0, t<0$ and $\varepsilon(y, t)=-y K_{o}, t \geq 0$. Each material element thus undergoes a step strain history, with the strain level increasing linearly with the distance of the material element from the centroid. By $E q$ 8, the reduced time becomes

$$
\xi(y, t)=\frac{t}{\phi\left(-y K_{o}\right)}
$$

The stress relaxation response is obtained from $\mathrm{Eq} 7$, which reduces to

$$
\sigma=-G\left[\frac{t}{\phi\left(-y K_{o}\right)}\right] y K_{o}
$$

Let $h$ denote the depth of the cross section. It is convenient to introduce the nondimensional coordinate $\bar{y}=y / h$ and, recalling $E q 3$, the nondimensional time $\bar{t}=t / \tau_{0}$. Note that the maximum strain in the beam is $\varepsilon_{\max }=h K_{o} / 2$. Equation 13 is now rewritten as

$$
\frac{\sigma}{G_{o}}=-2 \bar{y} G\left[\frac{\bar{t} \tau_{o}}{\phi\left(-2 \varepsilon_{\max } \bar{y}\right)}\right] \frac{\varepsilon_{\max }}{G_{o}}
$$

Note that when $G(t)$ is substituted from $E q 3, \tau_{0}$ drops out of (14).

If $\varepsilon_{\max }$ is sufficiently small, then $\phi \approx 1$ and the stress relaxation is essentially the same at all material elements. For larger values of $\varepsilon_{\max }$, the stress relaxes faster as the distance of the element from the centroid is increased. This is shown in Fig. 3, where results are calculated for $\varepsilon_{\max }=0.1$. Note that the 


\section{A. Wineman and R. Kolberg}

plots of $\sigma / G_{o}$ vs. $\bar{t}$ for larger $\bar{y}$ cross those for the material elements near the centroid. The implications of this are shown in Fig. 4 in the plots of $\sigma / G_{o}$ vs. $\bar{y}$ at various times. Although the stress increases linearly with $\bar{y}$ for small times, the stress distribution changes completely as time increases. The maximum stress no longer occurs at the outermost material element. Its location moves towards the centroid.

Suppose the beam has a rectangular cross section with base $b$ and height $h$. Then Eq 10 can be written as

$$
\frac{M}{M_{o}}=\frac{24}{G_{o}} \int_{0}^{1 / 2} \bar{y}^{2} G\left[\frac{\bar{t} \tau_{o}}{\phi\left(-2 \varepsilon_{\max } \bar{y}\right)}\right] d \bar{y}
$$

where $M_{o}=G_{o} I K_{o}$ and $I=b h^{3} / 12$. Plots of $M / M_{o}$ vs. $\bar{t}$ are shown in Fig. 5 for both linear viscoelastic $(C=0$ in $E q 5)$ and nonliner viscoelastic $(C=0.05)$ behavior. The bending moment relaxes much faster because of the nonlinear material behavior. Initially, the outer material elements initially contribute more to the bending moment than those closer to the centroid because they carry higher stresses and have larger moment arms about the z-axis. However, because of the accelerated stress relaxation at the outer material elements, their contribution to the bending moment rapidly diminishes. In other words, the bending moment decreases as a result of stress relaxation at all material elements as well as the rapid decrease in stress transmitting capacity of the outer material elements.

\section{CONSTANT CURVATURE RATE HISTORIES}

Suppose that the beam is bent so that the curvature increases at a constant rate. Then $K(t)=\alpha t$ and by $E q 11, \varepsilon(y, t)=-\alpha y t$. Each material element undergoes a constant strain rate history, and the rate

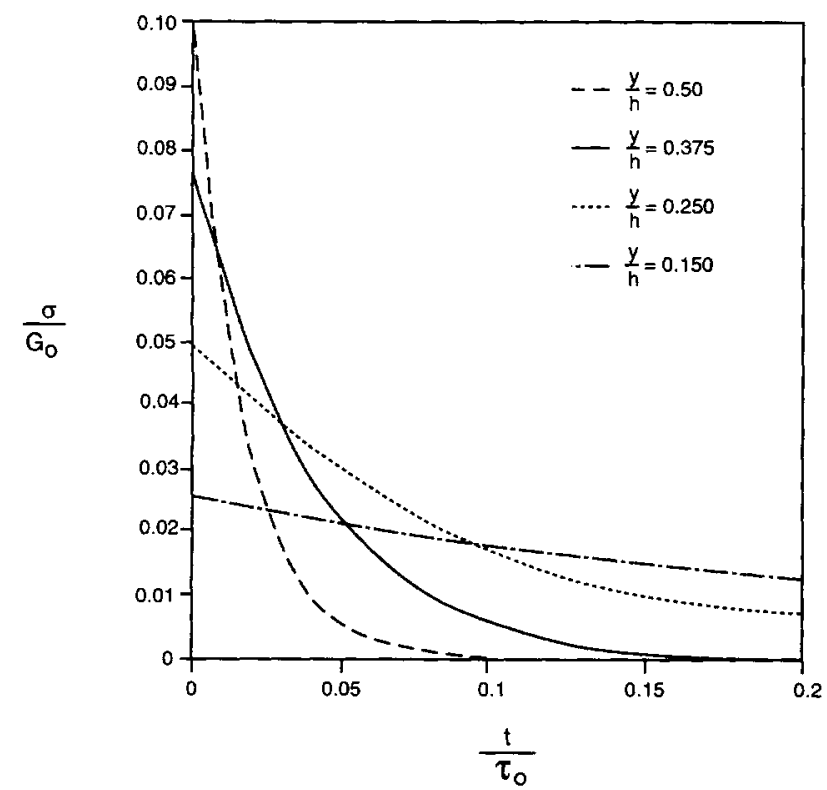

Fig. 3. Step curvature history $\left(K_{o}=0.2\right)$-stress histories at different positions.

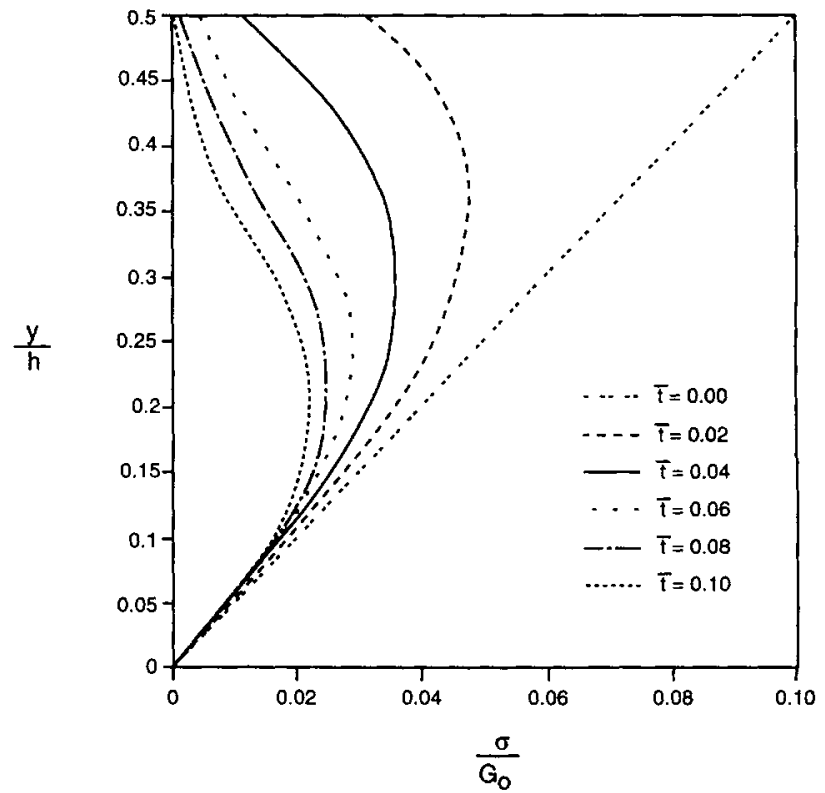

Fig. 4. Step curvature history $\left(K_{o}=0.2\right)$-stress distribut tions at different times.

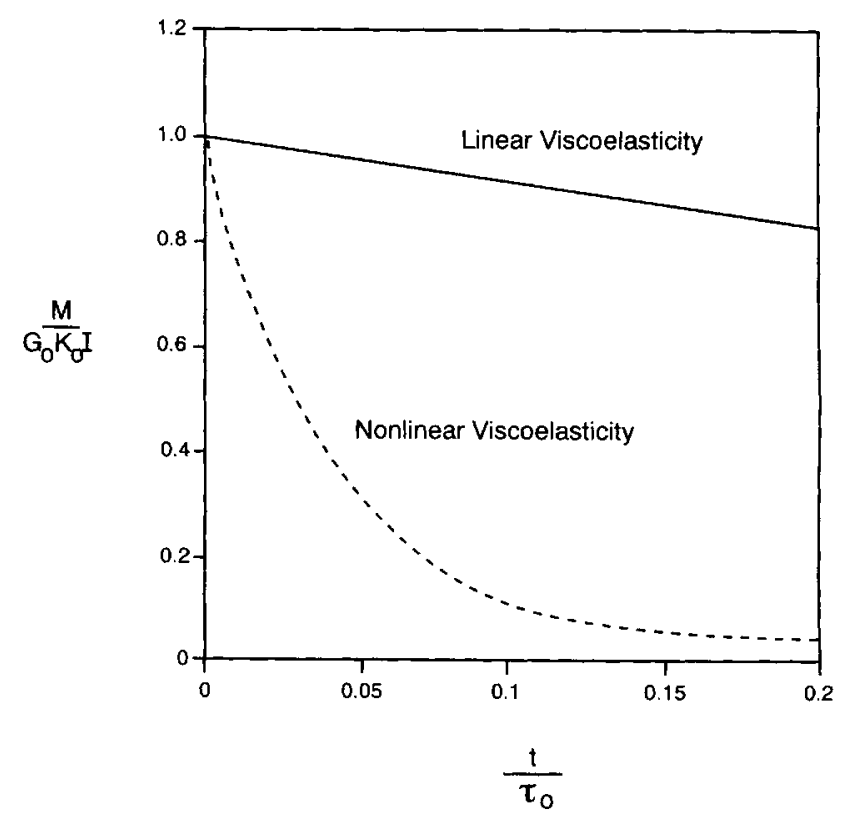

Fig. 5. Step curvature history $\left(K_{0}=0.2\right)$-bending moment histories for linear and nonlinear viscoelastic response.

increases with the distance of the material element from the centroid. Note that this is the strain history commonly used in uniaxial extension experiments. If the strain rate at a material element is high enough, the stress may reach a local maximum and then decrease, which is usually referred to as yield.

By $E q$, the reduced time $\xi(y, t)$ is

$$
\xi(y, t)=\int_{0}^{t} \frac{d x}{\phi(-y \alpha x)}
$$




\section{Mechanical Response of Beams of a Nonlinear Viscoelastic Material}

Let $\bar{t}$ and $\bar{y}$ denote the same non-dimensional variables as before, and introduce the additional nondimensional variables $\bar{\xi}=\xi / \tau_{o}$ and $\bar{\alpha}=h \alpha \tau_{\circ}$. Equation 16 becomes

$$
\bar{\xi}(\bar{y}, \bar{t})=\frac{1}{\bar{\alpha} \bar{y}} \int_{0}^{\bar{\alpha} \bar{y} t} \frac{d z}{\phi(-z)}
$$

The nondimensional form of the stress, from $E q 7$, is

$$
\frac{\sigma}{G_{0}}=-\frac{\bar{y} \bar{\alpha}}{G_{o}} \int_{0}^{t} G\left[\tau_{0}(\bar{\xi}(\bar{y}, \bar{t})-\bar{\xi}(\bar{y}, \bar{s}))\right] d \bar{s}
$$

in which $\bar{s}=s / \tau_{o}$. The bending moment, by Eq 10 is

$$
\frac{M}{M_{o}}=\frac{24}{G_{o}} \int_{0}^{1 / 2} \bar{y}^{2} \int_{0}^{t} G\left[\tau_{o}(\bar{\xi}(\bar{y}, \bar{t})-\bar{\xi}(\bar{y}, \bar{s}))\right] d \bar{s} d \bar{y}
$$

where $M_{o}=G_{o} I K_{o}$ and $K_{o}=\alpha \tau_{o}$.

Results are presented for $\bar{\alpha}=2.0$, which corresponds to a maximum strain of 0.1 when $\bar{t}=0.1$. Figure 6 shows plots of stress histories, $\sigma / G_{\circ}$ vs. $\bar{t}$ for material elements at several distances $\bar{y}$ from the centroid. At $\bar{y}=0.125$, the strain rate is small enough that the stress increases almost linearly with time. At $\bar{y}=0.250$, the strain rate is larger and there is some acceleration of stress relaxation. The plot of $\sigma / G_{o}$ vs. $\bar{t}$ begins to bend over. At $\bar{y}=0.375$ and beyond, the strain rate is large enough that the plots $\sigma / G_{o}$ vs. $\bar{t}$ have local maxima. Since the strain at each material element is proportional to time, the time axis can be re-scaled into a strain axis, thereby producing a stress-strain plot for each material element. Such plots for $\bar{y}=0.375$ and beyond have local maxima, which indicates yield. Thus, because the strain rates increase with distance from the centroid, there will be outer layers of material elements that are undergoing

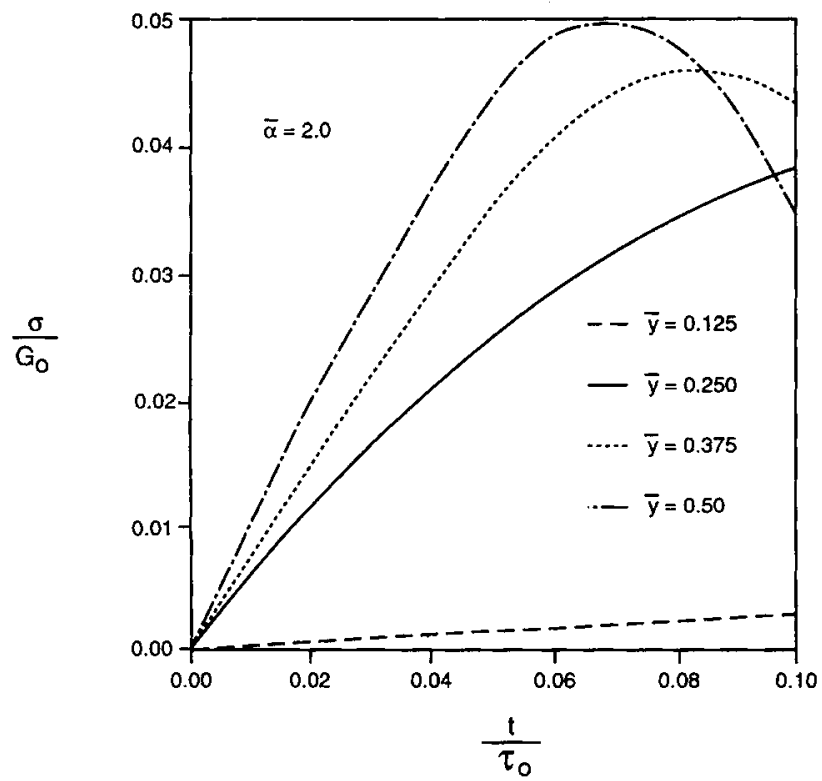

Fig. 6. Constant curvature rate history $(\bar{\alpha}=2.0)$-stress his tories at different positions. yield. The approach presented here provides a continuous transition to yield.

Stress distributions, $\sigma / G_{o}$ vs. $\bar{y}$, at various times are plotted in Fig. 7. Initially, the stress distribution is linear in $\bar{y}$. As time increases, this distribution becomes completely altered as the stress begins to decrease at the outer material elements. That is, the maximum stress on the cross section begins to decrease with time and its location moves into the beam. The effect of this on the bending moment is shown in Fig. 8. When the stress at the outer material elements begins to decrease with time, so does their contribution to the bending moment. Consequently, the bending moment vs. time plot also has a local maximum and begins to decrease with time.

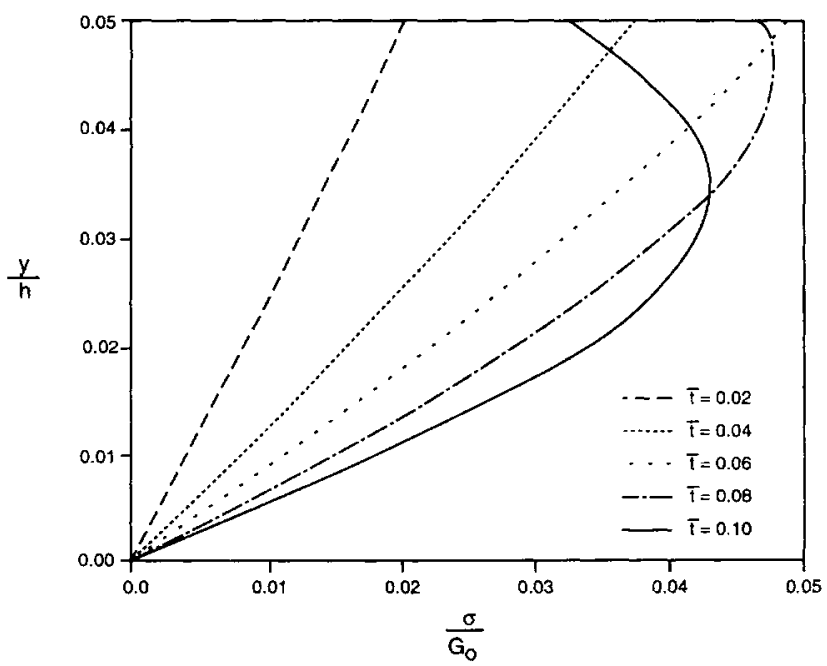

Fig. 7. Constant curvature rate history $(\bar{\alpha}=2.0)$-stress distributions at different times.

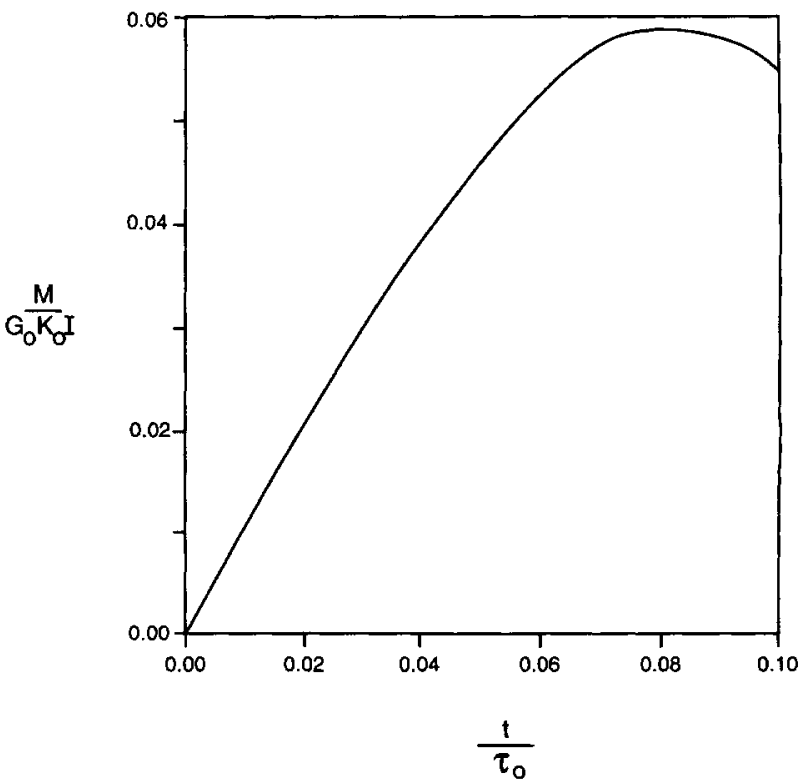

Fig. 8. Constant curvature rate history $(\bar{\alpha}=2.0)$-bending moment history. 


\section{CONCLUDING REMARKS}

Suppose that the line $y=0$ is not an axis of symmetry, or that the material displays different response in extension or compression. Then Eq 9 can be satisfied at each instant only if the location of the neutral axis changes with time, that is, only if $d(t) \neq$ 0 . Its motion will be determined by the spatial variation of stress relaxation. This will be studied in future work.

\section{REFERENCES}

1. R. M. Shay, Jr., and J. M. Caruthers, Adv. Rheology, Proc. IXth Intl Congress Rheology, 1, 549 (1984).

2. R. M. Shay, Jr., and J. M. Caruthers, J. Rheol, 30, 781 (1986).

3. W. G. Knauss and I. J. Emri, Comp. Struct., 13, 123 (1981).

4. W. G. Knauss and I. J. Emri, Polym. Eng. Sci., 27, 86 (1987).

5. A. S. Wineman and W. K. Waldron, Jr., Polym Eng. Sci., 33, 1217 (1993). 